\section{DISEÑO PARA EL CONOCIMIENTO REGLADO DE ACCESO LIBRE A TRAVÉS DE INTERNET}

\author{
M. C. Morillo Balsera \\ L. Sebastian Lorente \\ M. L. Casado Fuente \\ Ingeniería Topográfica y Cartografía, \\ Universidad Politécnica de Madrid \\ mariadelcarmen.morillo@upm.es \\ luis.sebastian@upm.es \\ ml.casado@upm.es
}

\begin{abstract}
The impact of information and communication technology, ICT, has opened a field of almost unlimited possibilities to communicate and share information. Between all the initiatives is important to point out the "OpenCourseWare (OCW)", movement of the OCW-MIT initiative. This movement provides a free, simple and consistent teaching materials for all non-profit educators, students and self-learners around the world. There are many reasons to believe that the improvement of open access to the creation and communication of information is good for human beings like in Floridi, 2002. This article includes several years of work in the teaching of algebra and geometry to engineers. All this is part of an invisible learning technology. Under this approach, seeks to create significant future applied mathematics, encouraging the creation, expansion and accessibility in an international environment like in Cobo and Moravec, 2011.
\end{abstract}

KEY WORDS: Technologies; TIC; OCW; OCW-MIT; invisible learning.

\section{INTRODUCCIÓN}

Las nuevas tendencias del aprendizaje relacionan tres procesos: la informatización de la sociedad, la globalización y las nuevas tecnologías, mediante los cuales las sociedades contemporáneas pueden adaptarse rápidamente a la sociedad del conocimiento. Estas sociedades tienen capacidad de generar, apropiar y utilizar el conocimiento para atender las necesidades de su desarrollo. De todos es conocido, que en Internet existe el mayor encuentro multicultural y se da la mayor coincidencia tecnológica de todos los tiempos como en Alonso y Gallego (2003). El principal problema que nos encontramos es el crecimiento, no coordinado, de esos recursos, con lo que se hacen bastante ineficaces las

\section{DESIGN FOR REGULATED OPEN ACCESS KNOWLEDGE THROUGH INTERNET}

RESUMEN: El impacto de las tecnologias de la información y la comunicación, TIC, ha abierto, un campo de posibilidades casi ilimitadas para comunicar y compartir la información. Entre las iniciativas creadas merece destacarse la de "OpenCourseWare (OCW)" movimiento de la iniciativa OCW-MIT, la cual proporciona un acceso libre, sencillo y coherente a los materiales docentes para educadores con carácter no lucrativo, a estudiantes y autodidactas de todo el mundo. Existen muchas razones para afirmar que la mejora del acceso libre a la creación y a la comunicación de la información, es positiva para los seres humanos como en Floridi, 2002. Este artículo, recoge varios años de trabajo en la enseñanza del Álgebra y la Geometría para ingenieros. Todo esto se enmarca dentro de la tecnología de un aprendizaje invisible. Bajo este enfoque, se busca la creación de futuros relevantes aplicados a las matemáticas, fomentando la creación, ampliación y accesibilidad en un entorno informacional como en Cobo y Moravec, 2011.

PALABRAS CLAVE: Tecnologías; TIC; OCW; OCW-MIT; aprendizaje invisible.

búsquedas generales. Por tanto es necesaria una cooperación invisible como en Fidalgo y Fernández (2009). Como ejemplo de todo lo anteriormente expuesto, está el espacio web OpenCourseWare (OCW) para promover el acceso libre y $\sin$ restricciones al conocimiento, ya que el potencial humano es universal. Recordemos que en abril de 2001, Massachusetts Institute of Technology, lanzó la iniciativa OCW-MIT con la visión de que las herramientas de enseñanza del aprendizaje de las principales instituciones del mundo, deberían ser de libre acceso a toda la humanidad: para estudiar, compartir y construir.

Esta iniciativa, ha tenido un gran impacto y ha impulsado a que otras Universidades de prestigio internacional se 
hayan adherido, generando proyectos propios en coordinación con OCW-MIT. Este es el caso de OpenCourseWare-Universia que, a través de medios digitales y en abierto, difunde los recursos docentes estructurados en asignaturas. En definitiva, se concibe como un escaparate para la difusión internacional de las actividades de nuestras Universidades.

Todo lo anterior está llevando a un nuevo paradigma del aprendizaje, lo que viene siendo Ilamado "el aprendizaje invisible", que no pretende proponer una teoría como tal, sino una metateoría capaz de integrar diferentes ideas y perspectivas. Por ello ha sido descrito como un protoparadigma, que se encuentra en fase beta y en plena etapa de construcción. En resumen, el aprendizaje invisible es una alternativa para ver el aprendizaje con otros ojos. Es: "aire fresco e independiente que circula por los pasillos de la educación", como en Cobo y Moravec (2011).

Se puede comprobar, que la gran mayoría de los materiales de los cursos de las asignaturas, que están en $0 \mathrm{CW}$, tienen en común los siguientes capítulos: programa de la asignatura, bibliografía, lectura obligatoria, lecturas complementarias, ejercicios, proyectos, prácticas, pruebas de evaluación, guía de aprendizaje y autores del material. Siendo no obligatorios la resolución de ejercicios, proyectos, así como las prácticas y las pruebas de evaluación. Además de los contenidos multimedia (sonidos, videos...), grupos de estudio (conexión con otros estudiantes interesados en este curso: colaborar en las tareas, hacerse preguntas acerca de los exámenes, o simplemente discutir los temas del curso) y herramientas o software especiales.

Como resumen, podemos reproducir el lema que aparece al abrir la página de MITOPENCOURSEWARE (Massachusetts Institute of Technology): "Unlocking Knowledge, Empowering Minds" ("Desbloqueo de conocimiento, empoderamiento de las mentes"). Así como algunas frases de sus profesores a destacar:

"My life is in teaching. To have a chance to do that with a world audience is just wonderful." (Gilbert Strang MIT Mathematics professor United States).

"OpenCourseWare is exactly the kind of thing that universities should be doing." (Larry Birenbaum, profesor del MIT y partidario $\mathrm{OCW}$ )
"My sense is that OCW will really enable us to create better educational linkages, and to fully integrate the learning experience." (Karen Willcox, profesora del MIT desde 2001)

El 4 de abril de 2011, el MIT celebró el 10. ${ }^{\circ}$ aniversario de la proclamación de MIT OpenCourseWare. Ahí se indicó que "en los últimos diez años, desde el anuncio en la primera página del New York Times, el MIT habian creado sitios web públicos de sus 2000 cursos y publicado materiales tales como: apuntes, boletines de problemas, planes de estudios de pregrado y postgrado de los 33 departamentos académicos del MIT, exámenes, simulaciones, incluso conferencias de videos, permitiendo a los educadores, estudiantes y aprendices independientes mejorar sus propias vidas y la vidas de quienes les rodean. En la próxima década, tienen la intención de ayudar a millones de personas más en todo el mundo para enseñar, aprender e innovar a través de nuestros recursos abiertos".

Hasta la fecha, se han publicado materiales de más de 2.000 cursos, que comprenden todos los departamentos académicos en el MIT. Han llegado a más de 100 millones de personas con los recursos educativos. Han inspirado a más de 250 Universidades de todo el mundo para compartir sus contenidos educativos. Sus materiales han ayudado a los educadores en Indonesia a mejorar sus cursos, a los empresarios en Haití en sus negocios, y a los estudiantes en África, con la confianza de que tienen acceso a la información mundial como en Oliveira, 2011.

Ante todo este desbloqueo del conocimiento que se viene produciendo y ante toda esta cooperación y aprendizaje invisible, aparece un nuevo empoderamiento como un proceso multidimensional de carácter social. Aquí, el liderazgo, la comunicación y los grupos autodirigidos, reemplazan la estructura piramidal mecanicista clásica por una estructura más horizontal, en donde la participación de todos y cada uno de los individuos dentro de un sistema, forman parte activa del control del mismo. Además de fomentar la riqueza y el potencial del capital humano, que posteriormente se verá reflejado, no solo en el individuo, sino también en la comunidad en la cual se desarrolla en Blanchard, Carlos y Randolph, 1997. 


\section{Objetivos}

Los objetivos que se pretenden en este artículo, tienen la inspiración y el seguimiento del MIT OPENCOURSEWARE, es decir trabajamos desde la filantropía y la difusión del conocimiento, dentro de las tecnologías del aprendizaje invisible como en Miyagawa, 2011.

Por tanto nuestros objetivos principales aplicados a las matemáticas están siendo:

1. Colaborar para la aplicación de todas las tecnologías que repercuten en la generación, difusión y utilización del conocimiento.

2. Ayudar a alumnos y profesores, mediante el aprendizaje invisible, para que el saber matemático sea más sencillo y atractivo.

\section{Desarrollo}

En los últimos cinco años, los profesores de Matemáticas de la ETSI de Topografía Geodesia y Cartografía de la UPM, hemos elaborado contenidos, para poner a disposición de nuestros alumnos, profesores y personas interesadas en aprender Matemáticas. La idea principal que se ha seguido, es que el conocimiento debe ser accesible a todo el mundo, encontrando en OCW la plataforma adecuada para alcanzar los objetivos propuestos.

La asignatura que se encuentra, en nuestro caso, en OPENCOURSEWARE de la Universidad Politécnica de Madrid es Álgebra y Geometría del Departamento de Ingeniería Topográfica y Cartografía. E.T.S.I. Topografía, Geodesia y Cartografía. Asignatura obligatoria de primer curso del Grado en Ingeniería en Geomática y Topografía, con 6 créditos (ECTS).

El diseño de la web de esta asignatura Álgebra y Geometría, comienza con los prerrequisitos y conocimientos previos que se necesitan para encarar con éxito los contenidos de dicha asignatura, recomendando algunas direcciones Webs, una de las cuales es un curso on-line de repaso y preparación en Matemáticas (repaso de Bachillerato), donde los autores de este artículo han participado. La dirección web es la siguiente:

$$
\text { http://moodle.topografia.upm.es/course/category.php?id=7. }
$$

Además, se da bibliografía preferente.

Posteriormente, se realiza una descripción general de la asignatura y los objetivos principales que se proponen conseguir con ella son:

1. Desarrollar la capacidad para la resolución de los problemas matemáticos que puedan plantearse en la ingeniería, desde este nivel de conocimiento.

2. Mejorar la aptitud para aplicar los conocimientos sobre álgebra lineal y geometría.

3. Enriquecer el razonamiento crítico.

Dentro del material docente desarrollado, hay que destacar la" Guía de aprendizaje".

\section{Guía de aprendizaje}

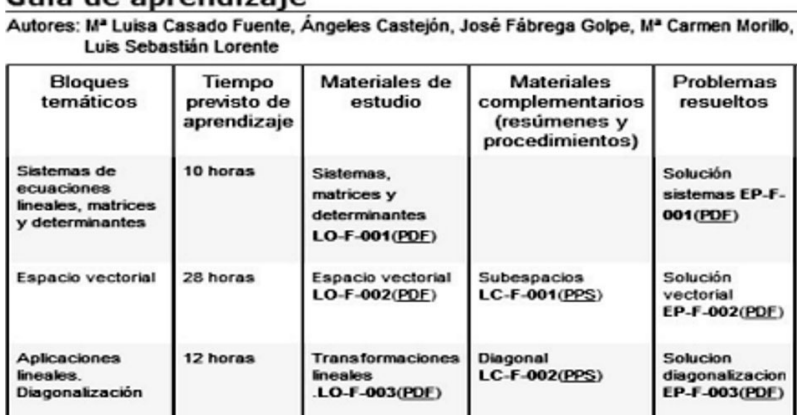

Figura 1. Visión incompleta de la Guía de aprendizaje de Algebra

$$
\text { y Geometría. }
$$

La "Guía de aprendizaje" es totalmente interactiva, proyectando una visión de conjunto de los contenidos y actividades, sirviendo al alumno para organizar su ritmo de aprendizaje y conteniendo los siguientes apartados:

- Bloques temáticos: son los temas en los que se divide la asignatura.

- Los tiempos previstos de aprendizaje: tiempo en horas que se deben dedicar a cada uno de los bloques temáticos o temas de la asignatura.

- Materiales de estudio donde se presentan los desarrollos de los contenidos teóricos con demostraciones y ejemplos resueltos en PDF de cada uno de los temas.

ARBOR Vol. 187 Extra 3 diciembre [2011] 177-182 ISSN: 0210-1963 
- Materiales complementarios (resúmenes y procedimientos), donde se presentan objetos de aprendizaje de contenidos teóricos, mediante presentaciones con metodología apropiada, como ayuda a la comprensión de conceptos matemáticos.

- Problemas resueltos, donde se presenta la resolución "paso a paso" de 422 problemas.

- Prácticas con DERIVE: resolución de problemas con el programa DERIVE. Las prácticas se presentan en PDF, son dirigidas, pudiéndose ver, directamente, la solución en la correspondiente casilla oculta o en el proceso completo con DERIVE. $\mathrm{A}=\left(\begin{array}{lll}1 & 0 & 0 \\ \mathrm{p} & 1 & 0 \\ 1 & 1 & 2\end{array}\right), \mathrm{B}=\left(\begin{array}{lll}2 & 2 & 1 \\ \mathrm{p} & 3 & 1 \\ 1 & 2 & 2\end{array}\right)$
- Exámenes: los exámenes se proponen por temas, lo que permite una evaluación continua, consistente en una serie de problemas cuya solución ha de consultarse tras su realización.

En las Figs. 2 y 3 se presenta un problema de diagonalización de matrices, para la resolución con Derive. Concretamente la Fig. 3 es el resultado después de hacer "clic" en cada una de las casillas de la Fig. 2.

También se realizan cuestionario de autoevaluación donde se recogen 524 preguntas, tipo test, con sus soluciones to-

3.- Estudiar, en función de los valores reales de p, la diagonalización de las matrices

\begin{tabular}{|l|l|l|l|}
\hline Matriz & $\begin{array}{l}\text { Polinomio } \\
\text { caracteristico }\end{array}$ & Valores propios & Subespacios propios \\
\hline A & & & \\
\hline B & & & \\
& & & \\
\hline
\end{tabular}

Figura 2. Presentación de problemas con DERIVE.

3.- Estudiar, en función de los valores reales de p, la diagonalización de las matrices

\begin{tabular}{|c|c|c|c|}
\hline Matriz & $\begin{array}{l}\text { Polinomio } \\
\text { característico }\end{array}$ & Valores propios & Subespacios propios \\
\hline $\mathrm{A}$ & $(2-w)(w-1)^{2}$ & $\begin{array}{l}2 \text { simple } \\
1 \text { doble }\end{array}$ & $\begin{array}{l}\text { Si } p \neq 0 \Rightarrow V_{w=1}=\{x=0, y=-\lambda, z=\lambda\} \Rightarrow \text { No } \\
\text { diagonalizable } \\
\text { Si } p=0 \Rightarrow V_{w-1}=\{x=-\alpha-\beta, y=\alpha, z=\beta\} \Rightarrow \\
\text { diagonalizable } \\
V_{w-2}=<\{(0,0,1)\}>\end{array}$ \\
\hline B & $\begin{array}{l}(1-w) \\
\left(w^{2}-6 w-2 p+7\right)\end{array}$ & $\begin{array}{l}\mathrm{w}_{1}=1 \\
\mathrm{w}_{2}=\sqrt{2(\mathrm{p}+1)}+3 \\
\mathrm{w}_{3}=-\sqrt{2(\mathrm{p}+1)}+3\end{array}$ & $\begin{array}{l}\text { (Debe especificar } \mathrm{p} \in(-1, \infty) \text { al hallar los } \\
\text { vectores propios) } \\
\mathrm{Si}-1<\text { p diagonalizable. } \\
\mathrm{Si} \mathrm{p} \leq-1 \text { No diagonalizable. }\end{array}$ \\
\hline
\end{tabular}

Figura 3. Resolución de problemas con DERIVE. 


\section{Sistemas, matrices y determinantes}

56.- Dada una matriz cualquiera $A \in M_{m \times n}$, el producto $\mathrm{AA}^{t}$ es siempre una matriz:

$\square$ a) inversible

$\mathrm{X}$ b) simétrica

$\square$ c) de dimensión $m \times n$

Una matriz es simétrica si coincide con su traspuesta $\left(\Lambda \Lambda^{t}\right)^{t}=\left(\Lambda^{t}\right)^{t} \Lambda^{t}=\Delta \Lambda^{\prime \prime}$

Unidad Docente de Matemáticas de la E.T.S.I.T.G.C. Siguiente

Figura 4. Diseño preguntas test. Opción correcta.

talmente explicadas. Las preguntas tipo test, se presentan de forma aleatoria con retroalimentación para la respuesta correcta, permitiendo así un aprendizaje activo.
Otro elemento a destacar es el "software propio", para la resolución de triángulos planos, esféricos y cálculos topográficos. Este permite al alumno proponer problemas de resolución de triángulos, así como comprobar los teoremas que se deben aplicar para la resolución de estos, además de ver los cálculos, paso a paso, en su resolución.

La Fig. 5. "Software TRES. Resolución problemas paso a paso", nos proporciona los teoremas aplicados (en este caso el teorema del coseno para lados) y los cálculos realizados (en este caso es la simple sustitución de los valores del triángulo esférico anteriormente mencionado). Señalamos que en la Fig. 5 se ve parte de la pantalla.

En definitiva, se ha creado un diseño interactivo de la asignatura para que el alumno adquiera competencias especificas y transversales en las materias objeto de estudio.

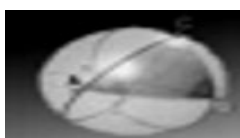

TRIÁNGULOS ESFÉRICOS

- Tres lados

- Tres áneulos

- Dos lados y el ángulo comprendildo

- Dos áneulias y el lado comprendilido

- Dos lados Y el ängulo opuesto a uno die ellios

- Dos áneulos y el lado operesto a uno de ellios

- Cálculo de la distancia
T. Planos T. Esfericos

\section{- Sexacesimal $O$ centesimal}

Teoremas aplicados.

Teorema del coseno para lados:

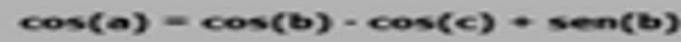

$\cos (b)-\cos (a)-\cos (c)-\operatorname{sen}(a)$

$\cos (c)-\cos (a)-\cos (b)+\operatorname{sen}(a)$

Calculos:

$\cos (a)-\cos \left(75^{\circ}-47^{\circ} 0^{2}\right) \cdot \cos (10)$

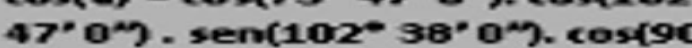

$a=93^{\circ} .4 * 44.45^{*}$

$\cos (B)-(\cos (b)-\cos (c)-\cos (a))$

Figura 5. Software TRES. Resolución problemas paso a paso. 


\section{Resultados y Conclusiones}

Uno de los resultados que consideramos más importantes es que a medida que vamos enriqueciendo nuestra versión "on line" de la asignatura Álgebra y Geometría, van mejorando los resultados en la calidad del aprendizaje.

También debemos señalar, que los profesores que participamos en la creación de la asignatura descrita en este artículo, desde que apostamos por fomentar el aprendizaje invisible, nos identificamos cada vez más con la reflexión de la profesora del MIT Karen Willcox, la cual manifiesta que "es la mejor experiencia educacional vivida en estos últimos años". Además, la mayoría de los docentes estamos convencidos en la necesidad de adecuar todo el sistema educativo a los tiempos actuales.
Por último comentar que el trabajo "on line" de esta asignatura, ha sido ganador de la IV edición de los Premios MEC-Universia dentro de la Iniciativa en OCW 2010. Esta distinción valora la calidad de los contenidos y los materiales publicados en la asignatura. El objeto de este concurso, como dicen sus bases, es el de promover la difusión en abierto, a través de Internet, de las propuestas docentes, estructuradas por asignaturas, que utilizan los profesores de las universidades españolas en el desarrollo de su actividad docente reglada de las titulaciones universitarias oficiales de grado o postgrado. Resaltando que el voto de los usuarios ha sido determinante para la concesión del premio, por lo que cabe pensar, que los contenidos de dicha asignatura están siendo accesibles, útiles e interesantes para la formación matemática en nuestros entornos universitarios.

\section{BIBLIOGRAFÍA}

Alonso, C. y Gallego, D. (Coord.) (2003): Informática y praxis educativa, UNED, Madrid.

Blanchard, K.; Carlos, J. y Randolph, A. (1997): Empoderamiento: 3 Claves para lograr que el proceso de facultar a los empleados funcione en su empresa, Norma S.A, Bogotá.

Cobo, C. y Moravec, W. J. (2011): "Aprendizaje invisible", Col/lecció Transmedia XXI, pp. 24-25, 113-114, Barcelona.

Fidalgo, A. y Fernández, F. (2009): "Cooperación Invisibe. El Proyecto María
(Métodos de apoyo y recursos interactivos de aprendizaje)", Arbor CLXXXV Extra, pp. 139-153, Madrid.

Floridi, L. (2002): "On the intrinsic value of information objects and the infosphere", Ethics and information technology, 4, pp. 287-304.

Miyagawa, S. (2011): Celebrating a Decade of Open Sharing: 2001-2011, MIT OpenCourseWare, Massachusetts.

Oliveira, C. (2011): Celebrating a Decade of Open Sharing: 2001-2011, MIT OpenCourseWare, Massachusetts. 